\title{
The runaway supergiant HD 188209 (O9.5Iab): a binary or a pulsating star?
}

\author{
Garik Israelian $^{1}$, Artemio Herrero ${ }^{1}$, E. Santolaya-Rey ${ }^{1}$, A. Kaufer ${ }^{2}$, \\ F. Musaev ${ }^{3}$, G. Galazutdinov ${ }^{3}$, and A. Galeev ${ }^{3,4}$ \\ ${ }^{1}$ Instituto de Astrofísica de Canarias, \\ E-38200 La Laguna, Tenerife, Spain \\ ${ }^{2}$ Landessternwarte Königstuhl, D-69117 Heidelberg, Germany \\ ${ }^{3}$ Special Astrophysical Observatory of the Russian AS, \\ Nizhnij Arkhyz 357147, Russia \\ ${ }^{4}$ Department of Astronomy, Kazan State University, \\ Kazan, Kremlevskaja Str., 420008, Russia
}

\begin{abstract}
We report radial velocity studies of photospheric absorption lines from spectral time series of the late O-type runaway supergiant HD 188209. Radial velocity variations with a quasi-period $\sim 2$ days have been detected in high-resolution echelle spectra and most probably indicate that the supergiant is pulsating. Night-to-night variations in the position and strength of the central emission reversal of the $\mathrm{H} \alpha$ profile have been observed. The fundamental parameters of the star have been derived using state-of-the-art plane-parallel and unified non-LTE model atmospheres, these last including the mass-loss rate. The binary nature of this star is not suggested either from Hipparcos photometry or from radial-velocity curves.
\end{abstract}

\section{Observations}

The observations have been carried out in different runs using the Coudé Echelle Spectrometer at the $1 \mathrm{~m}$ telescope of the $S A O$ (N. Caucasus, Russia). The spectra (45 in total) have a $\mathrm{s} / \mathrm{N} \geq 100$ per resolution element, and an average resolution $R=40000$ in the wavelength region $4400-7000 \AA$. The control measurements of interstellar Ca II and $\mathrm{NaI} D$ lines and of the DIB revealed a small scatter of the order of $0.8-1.5 \mathrm{~km} \mathrm{~s}^{-1}(1 \sigma)$. All stellar absorption lines exhibited variations about their respective mean velocities of at least 2-3 times the dispersion of the DIB velocities.

\section{Analysis}

The method followed in determining the stellar parameters from the spectrum using NLTE, plane-parallel hydrostatic model atmospheres has been described in detail by Herrero et al. (1992 and references therein). We obtained the parameters $T_{\text {eff }}=31500 \mathrm{~K} \pm_{500}^{1000}, \log g=3.0 \pm 0.1$ (adopting a microturbulent velocity of $\left.10 \mathrm{~km} \mathrm{~s}^{-1}\right)$ and $\epsilon=0.12 \pm 0.03(\epsilon=\mathrm{He} /(\mathrm{H}+\mathrm{He})$; the solar abundance is $\epsilon=0.09)$. Then we used a spherical, non-hydrostatic model atmosphere (Santolaya-Rey et al. 1997), in order to improve the already derived parameters and also to obtain 
the mass-loss rate from $\mathrm{H} \alpha$. We took the wind terminal velocity $1700 \mathrm{~km} \mathrm{~s}^{-1}$ from Haser (1995). An average logarithmic mass-loss rate for HD 188209 was derived between -6.0 and -5.7 . With the stellar parameters given above we can determine the radius, luminosity and mass of HD 188209 as described in Herrero et al. (1992). For $\mathrm{M}_{\mathrm{v}}=-6.0 \mathrm{mag}\left(M_{\mathrm{bol}}=-9.0 \mathrm{mag}\right)$ given by Howarth \& Prinja (1989), we obtain $R / \mathrm{R}_{\odot}=20.9 \pm 0.06, \log \left(L / \mathrm{L}_{\odot}\right)=5.59 \pm 0.16$ and $M / \mathrm{M}_{\odot}=16.6 \pm 0.22$.

\section{Discussion}

Our target belongs to the group of stars for which the existence of a compact companion has been proposed in the literature (Stone 1982). To establish the presence of a possible companion we have studied the radial velocities (RVs) of absorption lines by combining them in different groups. The highest peak in the Fourier power spectrum was always centered between frequencies 0.43 $0.45 \pm 0.05 \mathrm{~d}^{-1}$ (2.2-2.31 days). The velocity-excitation relationship found for many hot supergiants exists also in HD 188209. Our results suggests that deeper layers in the atmosphere pulsate with smaller amplitudes.

The average $\mathrm{H} \alpha$ profile consists of three components, a central emission accompanied by blue and red absorptions. The emission is not always centered exactly on the rest wavelength but is varying. A period analysis of the RV curves of the central emission resulted in the detection of quasi-periodic variability with a frequency $0.42 \pm 0.14 \mathrm{~d}^{-1}$ (2.35 days) which is identical to the one found from many photospheric lines.

It is difficult to interpret 2.2 days period as a result of the orbital motion in the binary system. A simple estimate shows that an $\mathrm{O}$ supergiant does not fit within the orbit. Its Roche radius would be less than the stellar radius. Even if it would fit, the tides in such a tight binary would be very strong making the star to speed up quickly until the rotation period (13 days) matches the orbit. The last argument comes from the relations between excitation energy and radial velocity amplitude and excitation energy and mean radial velocities. Later is a good discriminant between pulsations and Keplerian motions. In a binary system one would expect all lines to have the same amplitude of the radial velocity variations independent on their total excitation energy. The absence of any periodic signals in the Hipparcos photometric data is another argument against of the binary scenario.

\section{References}

Haser, S., 1995, Dissertation Universität München

Herrero, A., Kudritzki, R.P., Vilchez, J.M., Kunze, D., Butler, K., Haser, S. 1992, A\&A 261, 209

Howarth J., Prinja, R. 1989, ApJS 69, 527

Santolaya-Rey, E., Puls, J., Herrero, A. 1997, A\&A 323, 488

Stone, R. 1982, ApJ 261, 208 